【电子与信息科学 / Electronics and Information Science】

\title{
基于双层随机规划的云监控平台定价策略
}

\author{
赖朝安，侯延行 \\ 华南理工大学工商管理学院, 广东广州 510640
}

摘 要：定价是企业运营的关键决策，云服务作为新兴产业，目前还未形成统一的定价标准。针对云 监控服务平台的定价问题，分析云监控服务提供商与客户之间的利益关系，建立两阶段动态博弯模型，围 绕利润最大化目标提取出核心参数与变量，同时考虑设备故障与生产损失等方面的不确定性，构建出双层 随机规划模型，结合极点搜索思想给出相应的求解算法，并通过实例证明模型的有效性. 计算结果显示, 提供商利润随着客户数量的增加呈递增趋势，同时多类型的服务模式在一定程度上有助于服务提供商利润 的增加; 调节定价策略，让较大客户购买基础服务类型，剩余客户选择深层服务，往往可使得服务提供商 的利润达到最优.

关键词：工业工程；定价策略；云服务；云监控；双层规划；不确定性；动态博弯；利润最大化；极 点搜索

中图分类号：F224.1；C931.1 文献标志码：Aｄoi：10.3724/SP. J. 1249.2020.04433

\section{Pricing strategy of cloud monitoring platform based on bilevel stochastic programming}

\author{
LAI Chaoan and HOU Yanhang \\ School of Business Administration, South China University of Technology, Guangzhou 510640, Guangdong Province, P. R. China
}

\begin{abstract}
Pricing is a key decision of enterprise operation. As a new industry, the cloud service has not yet formed a unified pricing standard. Aiming at the pricing problem of cloud monitoring service platform, this paper analyzes the interest relationship between providers of cloud monitoring service and customers, establishes a two-stage dynamic game model, and extracts the core parameters and variables for the target of profit maximization. This paper also takes into account the uncertainty of equipment failure and production loss, and constructs a bilevel stochastic programming model, then a new algorithm combined with pole search method is proposed to find the global optinal solution. Finally, an example is given to demonstrate the model effectiveness. The computation results show that the profit of the provider is increasing with the increase of the number of customers. At the same time, the multi-type service mode will help the service provider increase its profit to a certain extent. By adjusting the pricing strategy, the platform makes most customers purchase the basic service type and the remaining customers choose the deep service, often optimizes the profits of service providers.
\end{abstract}

Received: 2019-06-16; Accepted: 2019-10-22

Foundation: Natural Science Foundation of Guangdong Province (2018A030313079) ; Soft Science Project of Guangdong Province (2019A101002006); The 13th Five-Year Plan for the Development of Philosophy and Social Sciences in Guangzhou (2018GZYB16)

Corresponding author: Associate professor LAI Chaoan. E-mail: chalai@ scut.edu.cn

Citation: LAI Chaoan, HOU Yanhang. Pricing strategy of cloud monitoring platform based on bilevel stochastic programming [ J $]$. Journal of Shenzhen University Science and Engineering, 2020, 37(4) : 433-440. (in Chinese) 
Key words : industrial engineering; pricing strategy; coloud service; cloud monitoring; bilevel programming; uncertainty; dynamic game; profit moximization; pole search

随着互联网时代技术的高速发展，出现了云服 务这种 IT 资源交付的新型商业模式，其具有资源 配置动态化、需求服务自助化、网络访问便捷化和 易扩展等诸多优点, 蕴含了极高的商业价值, 也不 断提高着企业对于资源的整合和利用能力 ${ }^{[1]}$ 。价格 影响着云平台的收益水平和资源利用效率，近些年 云计算服务的定价方法受到商界和学术界的广泛关 注. YOUSEFF 等 ${ }^{[2]}$ 提出单位定价 (per-unit pricing)、 分级定价 (tieredpricing) 和预订定价 ( subscriptionbased pricing) 3 种定价模式. CHUN 等 ${ }^{[3]}$ 从云服务 提供商的角度研究了按用量定价模型 (pay-per-use pricing model) 和认购定价模型 (subscription pricing model). 在这些静态定价机制下，云服务提供商单 方面决定价格，这种模式具有服务价格易管理、计 价稳定和有效性高等优势, 客户也易于理解和接 受, 因此在云服务发展初级阶段多被采用, 但随着 云服务市场规模的扩大，该模式无法解决有限资源 供给与不断增加的市场需求间的矛盾，在客户需求 趋于多元化与个性化的成熟阶段，出现严重的客户 流失现象，不利于云服务提供商的收人增加 ${ }^{[4]}$. 近 年来有学者开始研究可调整价格的动态计价模式, 如 MIHAILESCU 等 $^{[5]}$ 通过仿真对比了静态定价模式 和动态定价模式下的客户请求成功率、提供商资源 配置状态以及客户的平均福利, 发现动态定价模式 的 3 个参数均优于静态定价模式; YEO 等 ${ }^{[6]}$ 基于满 足客户应用和服务要求设计一种自动定价动态模 型，可使客户以未来的价格提前预定服务，有助于 客户未来的运营规划, 也提升了云服务提供商的利 润. 动态定价模型虽然理论上可实现利润的最大 化, 但现实环境存在较多的不确定情况, 影响动态 定价模式的有效性，SHARMA 等 ${ }^{[7]}$ 基于期权定价理 论提出一种风险可调节的云定价模型，考虑了财务 风险和交易对手风险，一定程度提升了定价的适用 性. 但是, 上述模型多是围绕供给方开展的研究, 基于资源占用、运营成本和资源供求关系等因素制 定价格，对客户侧的交互作用考虑较少.

为此, 本研究从提供商和客户双边的角度出 发, 通过双层随机规划模型建立定价机制, 实现提 供商与客户双方利润最大化的需求，所建模型涵盖 了现实中的不确定因素, 可确保云服务提供商在不
同环境下的收益.

\section{1 问题描述及假设}

\section{1 云监控服务现状}

研究对象为设备云监控服务提供商，服务费以 单台生产设备的年费形式收取，首年免费试用，到 期后需续费使用. 客户使用服务前，需在设备端安 装传感器采集设备数据, 提供商对数据进行云端监 控，发现异常立即反馈，维修工程师再根据反馈信 息进行预知维修，可有效降低客户生产线设备停机 率，减少停机造成的生产损失.

提供商给客户提供基础服务和深层服务 2 种可 选服务. 在基础服务类型中，云服务提供商仅为客 户提供监控平台，客户端设备传感器的建设费用和 维修费用等均由客户承担，此类服务年费较低; 在 深层服务类型中，云服务提供商承包不仅提供传感 器网络与数据传输网络等客户端建设, 还承接客户 企业的设备监控维修部门及人员外包服务，即客户 将设备监控维修工作全部交付于服务提供商, 在此 类服务下提供商就故障停机率的降低值 $\xi$ 预先给出 承诺值 $r$, 若未来一年实施中未达到此承诺值，提供 商需向客户赔偿，赔偿金额 $\delta$ 根据次年的 $\xi$ 与 $r$ 之间 的差距 $\eta$ 给出，由于次年的故障停机率未知，所以 $\xi 、 \eta$ 和 $\delta$ 都具有不确定性.

本研究从经济学中的经济人假设出发, 各方均 以实现自身利益最大化为目标，且具有独立的决策 权. 试用期结束后，云服务提供商掌握客户的信 息，可构建客户的利润函数，而客户并不知提供商 的经营数据，信息存在不对称性，且提供商占据优 势地位. 同时，提供商的盈利额与客户量紧密关 联，高度依赖于客户的决策，当提供商给出某种定 价后，客户会根据自身利益函数做出选择，决定是 否购买服务以及购买哪种服务，而提供商又可根据 客户的行为改变定价策略. 可见，这个重复动态博 弯问题具有 Stackelberg 特征：提供商利益函数包含 客户决策变量，客户利益函数中亦包含提供商决策 变量; 提供商具有优先决策权, 客户对提供商的定 价策略会做出反应决策，各客户决策相互独立，提 供商无法干涉任何客户的决策，但可通过构建客户 
的利润函数，提前预判各定价策略下客户的决策情 况，进而持续调整定价策略.

双层规划可较好地模拟层次性与递阶性，是解 决此类问题的有效方法. $\mathrm{HU}$ 等 ${ }^{[8]}$ 在政府与煤矿企 业的资源分配中引人双层规划理论，优化了矿业配 额方案; ZHANG 等 ${ }^{[9]}$ 用双层规划模型为作物种植 面积和水量分配问题提供了决策建议; KARIMI 等 ${ }^{[10]}$ 分析了电力能源的市场现状，并借助双层规 划解决了电力市场的实时定价问题; BENITA 等 ${ }^{[11]}$ 在投资组合管理领域引人双层规划，使投资策略尽 可能小地规避风险从而获得较高的利润. 以上研究 结果证明了双层规划解决动态博弯问题的有效性. 本研究结合双层规划思想与云服务行业的特点, 在 加人随机参数做出改进后进行模型的建立.

\section{2 常规双层规划模型}

常规双层规划模型为

$$
\begin{cases}\min _{x \in X} F(x, y) & \text { s.t. } G(x, y) \leqslant 0 \\ \min _{y \in Y} f(x, y) & \text { s.t. } g(x, y) \leqslant 0\end{cases}
$$

其中, $X$ 和 $Y$ 分别为上层和下层决策变量的集合; $F(x, y)$ 和 $f(x, y)$ 分别为上层和下层规划的目标函 数, $x$ 和 $y$ 分别为上层和下层规划的决策变量; $G(x, y)$ 和 $g(x, y)$ 分别为上层和下层规划的约束 条件, $G(x, y) \leqslant 0$ 和 $g(x, y) \leqslant 0$. 由式 (1) 可见,本 研究正是求解一个一主多从的双层规划问题.

\section{2 模型建立}

\section{1 决策变量}

$f_{\mathrm{c}}^{\prime}$ 为客户购买平台深层服务类型时, 单台设备 收取的年费; $f_{\mathrm{c}}$ 为客户购买平台基础服务类型时, 单台设备收取的年费; $x_{i}$ 为客户是否购买平台的服 务, 1 为买, 0 为不买; $m_{i}$ 为企业购买平台的服务 类型, 1 为深层服务类型, 0 为基础服务类型.

\section{2 参 数}

设 $I$ 为客户总数, $i$ 为以客户拥有的待监控设备 数量为指标, 按照降序排列的客户编号, 即 $i=1$, $2, \cdots, I, k(i)$ 为客户 $i$ 拥有的待监控设备数量, AXTELL $^{[12]}$ 认为, 企业规模服从幕律分布, 即 $k(i)=$ $c i^{-r}, c$ 为控制缩放比例的常量, $r>0 ; C_{\mathrm{c}}(i)$ 为客户 $i$ 的传感器建设费用, 企业规模与传感器建设数量 存在线性关系, 故 $C_{\mathrm{c}}(i)=e_{11} k(i)+e_{12}$, 其中, $e_{11}$ 和 $e_{12}$ 为线性函数的常量参数; $C_{\mathrm{c}}$ 为所有客户的传感
器建设费用之和, 即 $C_{\mathrm{c}}=\sum_{i=1}^{I}\left(e_{11} k(i)+e_{12}\right) ; C_{\mathrm{p}}$ 为 云提供商数据中心和监控中心的建设成本，由基础 服务类型的费用 $C_{\mathrm{p}(\mathrm{b})}$ 和深层服务的费用 $C_{\mathrm{p}(\mathrm{d})}$ 组成, $C_{\mathrm{p}}$ 与总客户数量有关, 其中单个客户建设成本与客 户规模存在线性关系, 则 $C_{\mathrm{p}}=e_{21} \sum_{i=1}^{I}\left(k(i) x_{i}+\right.$ $\left.e_{22}\right), e_{21}$ 和 $e_{22}$ 为常量参数; $R_{\text {loss }}(i)$ 为客户 $i$ 购买监 控云服务后所降低的生产损失额，同样与企业规模 存在线性关系, 则 $R_{\text {loss }}(i)=e_{31} k(i)+e_{32}, e_{31}$ 和 $e_{32}$ 为常量参数; $F_{\text {worker }}(i)$ 为客户 $i$ 在未购买深层服务 时的维修工程师年薪，与企业规模存在幂函数关 系, 则 $F_{\text {worker }}(i)=e_{41} k(i)^{e_{42}}, e_{41}$ 为比例系数, $e_{42}$ 为 幂函数常量参数; $F_{\text {worker }}$ 为所有客户在未购买深层服 务时的维修工程师年薪, $F_{\text {worker }}=\sum_{i=1}^{I} e_{41} k(i)^{e_{42}}$; $F_{\text {worker }}^{\prime}(i)$ 为客户 $i$ 购买深层服务后的维修工程师年 薪, $F_{\text {worker }}^{\prime}(i)=e_{51} k(i)^{e_{52}} ; F_{\text {worker }}^{\prime}$ 为所有客户购买深层 服务后的总维修工程师年薪, $F_{\text {worker }}^{\prime}=\sum_{i=1}^{I} e_{51} k(i)^{e_{52}}$; $\delta(i)$ 为平台对购买深层服务的客户 $i$ 所支付的赔偿 金费用，即

$$
\delta(i)= \begin{cases}\eta_{i} \times O_{i}, & \xi_{i} \leqslant r_{i} \\ 0, & \xi_{i}>r_{i}\end{cases}
$$

其中, $\eta_{i}$ 为客户 $i$ 现实故障率降低值与对其承诺值的 差额; $O_{i}$ 为客户 $i$ 相关设备产出额; $r_{i}$ 为对客户 $i$ 的 承诺降低值; $\xi_{i}$ 为客户 $i$ 的现实故障率降低值; $\delta$ 为 平台支付的总赔偿金，即

$$
\delta=\sum_{i=1}^{I} x_{i} m_{i} \eta_{i} O_{i}
$$

\section{3 基于双层随机规划的模型构建}

上层规划：提供商为两种类型的云服务年费定 价, 使自身利润实现最大化. 上层决策变量为 $f_{\mathrm{c}}^{\prime}$ 和 $f_{\mathrm{c}}$, 则平台基础服务板块的利润函数为

$$
g_{1}\left(x_{i}, m_{i}, f_{i \leq I \in \mathrm{N}^{+}}^{\prime}, f_{\mathrm{c}}\right)=\sum_{i=1}^{I} x_{i}\left(1-m_{i}\right) f_{\mathrm{c}} k(i)-C_{\mathrm{p}(\mathrm{b})}
$$

平台深层服务板块的利润函数为

$$
\begin{aligned}
g_{2}\left(x_{i}, m_{i}, f_{\mathrm{c}}^{\prime}, f_{\mathrm{c}}\right)= & \sum_{i=1}^{I} x_{i} m_{i} f_{\mathrm{c}}^{\prime} k(i)-C_{\mathrm{p}(\mathrm{d})}- \\
& C_{\mathrm{c}}-F_{\text {worker }}^{\prime}-\sum_{i=1}^{I} x_{i} m_{i} \eta_{i} O_{i}
\end{aligned}
$$

上层目标函数为

$$
\begin{aligned}
\max F\left(x_{i}, m_{i}, f_{\mathrm{c}}^{\prime}, f_{\mathrm{c}}\right)= & g_{1}\left(x_{i}, m_{i}, f_{\mathrm{c}}\right)+ \\
& g_{2}\left(x_{i}, m_{i}, f_{\mathrm{c}}^{\prime}\right)
\end{aligned}
$$


上层约束条件为

$$
\begin{gathered}
g_{1}\left(x_{i}, m_{i}, f_{\mathrm{c}}\right) \geqslant 0, g_{2}\left(x_{i}, m_{i}, f_{\mathrm{c}}^{\prime}\right) \geqslant 0, \\
f_{\mathrm{c}}^{\prime} \geqslant 0, f_{\mathrm{c}} \geqslant 0
\end{gathered}
$$

下层规划：每个客户做出是否购买的决策，实 现自身利润最大化. 下层决策变量为 $x_{i}$ 和 $m_{i}$. 客户 不购买任何服务类型的利润提升值为 0 . 客户购买 深层服务类型的利润提升函数为

$$
\begin{array}{r}
h_{i}^{\prime}\left(x_{i}, m_{i}, f_{\mathrm{c}}^{\prime}, f_{\mathrm{c}}\right)= \\
R_{\text {loss }}(i)+\eta_{i} O_{i}+ \\
F_{\text {worker }}(i)-f_{\mathrm{c}}^{\prime} k(i)
\end{array}
$$

客户购买基础服务类型的利润提升函数为

$$
h_{i}^{\prime}\left(x_{i}, m_{i \leq I \in \mathrm{N}^{+}}, f_{\mathrm{c}}^{\prime}, f_{\mathrm{loss}}(i)-f_{\mathrm{c}} k(i)-C_{\mathrm{c}}(i)\right.
$$

下层目标函数为

$$
\begin{gathered}
\max f_{i}\left(x_{i}, m_{i \leqslant I \in \mathrm{N}^{+}}, f_{\mathrm{c}}^{\prime}\right)=x_{i} m_{i} h_{i}^{\prime}\left(x_{i}, m_{i}, f_{\substack{i \in I \\
\mathrm{c}}}^{\prime}, f_{\mathrm{c}}\right)+ \\
x_{i}\left(1-m_{i}\right) h_{i}\left(x_{i}, m_{i \leqslant I \in \mathrm{N}^{+}}, f_{\mathrm{c}}^{\prime}, f_{\mathrm{c}}\right)
\end{gathered}
$$

\section{3 算例求解}

\section{1 模型初始化}

针对某精细化工装备制造公司进行数据采集和 模型求解. 该公司开发的云服务平台可对世界范围 内的客户工厂进行云监控, 平台于 2017 年上线, 首年内 12 家客户免费使用该平台，不同程度地降 低了生产损失，并表示服务年费合理将继续使用. 云监控平台运行机制请扫描论文末页右下角二维码 见图 $\mathrm{S} 1$.

12 个客户的信息包括设备数量 $k(i)$ 、相关设备

\begin{tabular}{|c|c|c|c|c|c|c|c|c|}
\hline \multirow{2}{*}{$i$} & \multirow{2}{*}{$k(i) /$ 台 } & \multirow{2}{*}{$10^{-6} \mathrm{O} /$ 美元 } & \multirow{2}{*}{$D / \%$} & \multirow{2}{*}{$10^{-6} L /$ 美元 } & \multicolumn{2}{|c|}{ 维修工程师数量 } & \multirow{2}{*}{ S/Gbyte } & \multirow{2}{*}{$10^{-6} C_{\mathrm{c}}(i) /$ 美元 } \\
\hline & & & & & $N_{\mathrm{b}}$ & $N_{\mathrm{a}}$ & & \\
\hline 1 & 135 & 321 & $3.5-1.2=2.3$ & 7.38 & 75 & 49 & 257.9 & 0.32 \\
\hline 2 & 77 & 106 & $4.8-1.9=2.9$ & 3.07 & 52 & 30 & 147.1 & 0.20 \\
\hline 3 & 55 & 95 & $4.2-1.8=2.4$ & 2.28 & 40 & 26 & 105.1 & 0.16 \\
\hline 4 & 48 & 86 & $6.4-3.5=2.9$ & 1.87 & 37 & 25 & 91.7 & 0.14 \\
\hline 5 & 42 & 81 & $9.5-5.9=3.6$ & 2.49 & 34 & 24 & 80.2 & 0.13 \\
\hline 6 & 31 & 62 & $7.2-4.2=3.0$ & 1.86 & 32 & 23 & 59.2 & 0.11 \\
\hline 7 & 25 & 55 & $5.8-3.0=2.8$ & 1.54 & 28 & 20 & 47.8 & 0.10 \\
\hline 8 & 21 & 32 & $4.9-2.8=2.1$ & 0.67 & 26 & 19 & 40.1 & 0.10 \\
\hline 9 & 16 & 28 & $4.3-1.6=2.7$ & 0.76 & 24 & 17 & 30.6 & 0.09 \\
\hline 10 & 13 & 21 & $7.9-4.6=3.3$ & 0.69 & 21 & 15 & 24.8 & 0.07 \\
\hline 11 & 11 & 12 & $8.2-4.9=3.3$ & 0.40 & 16 & 11 & 21.0 & 0.07 \\
\hline 12 & 10 & 9 & $8.9-5.7=3.2$ & 0.28 & 14 & 10 & 19.1 & 0.07 \\
\hline
\end{tabular}
年产出额 $O$ 、停机率的降低值 $D$ 、生产损失额 $L(L=$ $O \times D)$ 、购买深层服务前和购买深层服务后的维修 工程师数量 $N_{\mathrm{b}}$ 和 $N_{\mathrm{a}}$ 、每年占用的存储空间 $S$ 和建设 客户的传感器费用 $C_{\mathrm{c}}(i)$ 见表 1 .

表 1 客户信息统计

Table 1 Customer information table

由表 1 数据拟合得到 2.2 节中对应的函数方程

$$
\begin{aligned}
& k(i)=137.4 i^{-0.864} \\
& C_{\mathrm{c}}(i)=0.002 k(i)+0.050 \\
& R_{\mathrm{loss}}(i)=0.052 k(i)-0.165
\end{aligned}
$$

阿里云 2017 年价格为每 80 Gbyte 存储空间 948 美元/年, 客户总占用空间为 924.6 Gbyte, 由于最 小购买单位为 80 Gbyte，所以需购买 960 Gbyte，即 存储空间成本为 11376 美元/年，相关软件使用年 限为 10 年, 传感器使用年限为 3 年, 则

$$
\begin{aligned}
C_{\mathrm{p}}= & e_{21} \sum_{i=1}^{I} k(i) x_{i}+e_{22}= \\
& 0.024 \sum_{i=1}^{I} k(i)+0.012
\end{aligned}
$$

技术工人年薪为 $24 \times 10^{3}$ 美元/年，根据表 1 中 维修工程师数量，可获取购买深层服务前后的薪资 曲线函数分别为

$$
\begin{aligned}
& F_{\text {worker }}(i)=0.024 \times 4.052 \times k(i)^{0.5890} \\
& F_{\text {worker }}^{\prime}(i)=0.024 \times 3.409 \times k(i)^{0.5289}
\end{aligned}
$$


实例的双层随机规划模型为

$$
\begin{aligned}
& \max F\left(x_{i}, m_{i}, f_{\mathrm{c}}^{\prime}, f_{\mathrm{c}}\right)=\sum_{i=1}^{I} x_{i} m_{i} f_{\mathrm{c}}^{\prime} k(i)+\sum_{i=1}^{I} x_{i}\left(1-m_{i}\right) f_{\mathrm{c}} k(i)-\frac{0.024 \sum_{i=1}^{I} x_{i} k(i)+0.012}{10}- \\
& 0.024 \sum_{i=1}^{I} 3.409 k(i)^{0.5298}-\frac{\sum_{i=1}^{I} x_{i} m_{i}[0.002 k(i)+0.05]}{3}-\sum_{i=1}^{I} x_{i} m_{i} \eta_{i} O_{i} \\
& \sum_{i=1}^{I} x_{i}\left(1-m_{i}\right) f_{\mathrm{c}} k(i)-\frac{0.024 \sum_{i=1}^{I} x_{i} k(i)+0.012}{10 \sum_{i=1}^{I} x_{i}} \geqslant 0 \\
& \sum_{i=1}^{I} x_{i} m_{i} f_{\mathrm{c}}^{\prime} \times 1.374 i^{-0.864}-\frac{0.024 \sum_{i=1}^{I} x_{i} k(i)+0.012}{10 \sum_{i=1}^{I} x_{i}}-\frac{0.002 k(i)+0.05}{3}- \\
& 0.024 \sum_{i=1}^{I} x_{i} m_{i} f_{\mathrm{c}}^{\prime} \times 3.409 \times k(i)^{0.5298}-\eta_{i} \sum_{i=1}^{I} x_{i} m_{i} f_{\mathrm{c}}^{\prime} O_{i} \geqslant 0 \\
& \max f_{i}\left(x_{i}, m_{i}, f_{\mathrm{c}}^{\prime}, f_{\mathrm{c}}\right)=x_{i} m_{i}\left[0.052 k(i)-0.165+4.052 k(i)^{0.5890}-f_{\mathrm{c}}^{\prime} k(i)\right]+ \\
& x(i)\left(1-m_{i}\right)\left[0.052 k(i)-0.165-f_{\mathrm{c}} k(i)-0.002 k(i)+0.050\right] \\
& k(i)=137.4 i^{-0.864} \\
& f_{\mathrm{c}}^{\prime} \geqslant 0, f_{\mathrm{c}} \geqslant 0, x_{i}=0,1 ; m_{i}=0,1 ; i \leqslant I \in \mathrm{N}^{+}
\end{aligned}
$$

\section{2 不确定性的处理}

式(17) 和式(19) 中含有不确定的变量 $\xi_{i}$, 为消 除此不确定性, 可转换为常规双层规划问题进行 求解.

在上层规划中, 先将随机变量 $\xi$ 转换为期望 值, 再在期望约束下, 寻找解集使目标函数的期望 值达到最优. 本研究涉及的故障数据大多呈现泊松 分布特征, 假定工程师的维修效率无较大波动, 则 可将客户设备的停机率降低值 $\xi$ 近似按照服从泊松 分布计算, 记为 $\xi \sim P\left(r_{i}\right)$, 令 $P_{r}\left(\xi_{i}=\xi_{i j}\right)=\theta_{i j}$. 在 上层目标函数和约束条件中, 以 $\xi_{i}$ 为变量的函数都 是线性的, 由期望值算子性质可知, 用随机变量的 期望计出的函数值为函数的期望, 即可用 $E[\delta(i)]$ 代替原来的 $\delta(i)$ 构造各个期望函数, 则

$$
E[\delta(i)]=\sum_{\xi_{i} \leqslant r_{i}}\left(r_{i}-\xi_{i j} \theta_{i j}\right) \times O_{i}
$$

对于下层规划, 当 $\xi<r$ 时, 客户通过云监控 服务所降低的生产损失未达到预期, 但可获得赔 偿, 等效于降低值达到了 $r$; 而当 $\xi>r$ 时, 客户获 得比预期承诺更好的收益. 因此, 对客户而言, 不
确定情况下的最低收益即提供商给出的 $r$ 所对应的 生产损失降低额 $R_{\mathrm{loss}}(i)_{r}$. 在客户风险喜好未知的情 况下, 为提高模型鲁棒性, 本研究采用承诺值所对 应的 $R_{\mathrm{loss}}(i)_{r}$ 计算 $R_{\mathrm{loss}}(i)+\eta_{i} O_{i}$, 则可在不确定情 况下, 获取客户确定的决策, 保证下层规划解的唯 一性, 避免因下层决策变动对上层规划最优解造成 的影响.

\section{3 模型的求解}

双层规划的求解比较复杂, 因为即使最简单的 双层线性规划也是非确定性多项式困难 (nondeterministic polynomially hard, NP-hard) 问题, 不存在多 项式时的求解算法. 目前常用的解法包括极点搜索 法、分支定界法、罚函数法、下降方向法和智能优 化算法等 ${ }^{[13-14]}$, 但都不具普适性, 各有优缺点, 其 中下降方向法和智能优化算法获得的解并不能保证 是全局最优的, 有可能过早地收玫至局部; 罚函数 法虽可获取全局最优解, 但构造惩罚项较复杂; 极 点搜索法与分支定界法尽管在处理复杂度较高的双 层规划时计算量较大, 但在解决中小规模的双层规 划时效果较好, 此模型中的下层规划可行域为 0 或 
1 的点集, 极点搜索法的计算量会低于分支定界法, 因此,本研究采用极点搜索法思想对模型进行求解.

CANDLER 等 ${ }^{[15]}$ 指出，若双层线性规划问题的 全局最优解个数有限, 则其全局最优解一定能在约 束区域的某个极点处取得. 本研究模型中包含多个 下层从者规划, 各规划的顶点形成了顶点组合集, 因此, 若双层规划最优解存在, 必会出现在顶点组 合集中的某一个组合上，其中下层规划约束域为 0 或 1 的集合，顶点数较少，且从者规划之间有较强 的相关性，使得组合集数也受限，很容易在短时间 内搜索出最优解. 记双层规划问题的约束域为

$$
\begin{aligned}
\Omega= & \left\{\left(x_{i}, m_{i}, f_{\mathrm{c}}^{\prime}, f_{\mathrm{c}}\right) \mid g_{1}\left(x_{i}, m_{i}, f_{\mathrm{c}}\right) \geqslant 0,\right. \\
& g_{2}\left(x_{i}, m_{i}, f_{\mathrm{c}}^{\prime}\right) \geqslant 0, f_{\mathrm{c}}^{\prime} \geqslant 0, f_{\mathrm{c}} \geqslant 0, \\
& \left.x_{i}=0,1 ; m_{i}=0,1 ; i \leqslant I \in \mathrm{N}^{+}\right\}
\end{aligned}
$$

对任意给定的下层解集 $x_{i}, m_{i} \in \Omega$, 上层规划 问题的合理反应集为

$$
\begin{aligned}
Q= & \left\{f_{\mathrm{c}}^{\prime}, f_{\mathrm{c}} \mid\left(f_{\mathrm{c}}^{\prime}, f_{\mathrm{c}}\right) \in\right. \\
& \left.\arg \max f\left(x_{i}, m_{i}, f_{\mathrm{c}}^{\prime}, f_{\mathrm{c}}\right), f_{\mathrm{c}}^{\prime}, f_{\mathrm{c}} \in \Omega\right\}
\end{aligned}
$$

当下层 $I$ 个客户决策使自身目标函数达到最优 时, 由这多个决策值 $\left(x_{i}, m_{i}\right)$ 组成的最优解集合记 为 $\left(x_{I}, m_{I}\right)^{*}$, 其对应的多个 $\left(f_{\mathrm{c}}^{\prime}, f_{\mathrm{c}}\right) \in \Omega$ 组成了反 应集 $Q_{n}$, 模型中每个下层解集与上层决策变量存在 一对多的映射关系，即客户群的某种决策组合会在 平台的多个定价方案下保持不变. 据此, 可将 $\Omega$ 分 割成多个包含单一的 $\left(x_{I}, m_{I}\right){ }^{*}$ 与系列的 $\left(f_{\mathrm{c}}^{\prime}, f_{\mathrm{c}}\right) \in$ $Q_{n}$ 区域, 这样便将下层不定的决策变量 $\left(x_{i}, m_{i}\right)$ 在 $\left(f_{\mathrm{c}}^{\prime}, f_{\mathrm{c}}\right) \in Q_{n}$ 内化为确定项, 双层规划被转换为 $n$ 个线性规划问题, 每个线性规划的最优解记为 $\left(f_{\mathrm{c}}^{\prime}, f_{\mathrm{c}}, x_{I}, m_{I}\right){ }^{*}$. 这些最优解是模型在可行域不同 位置的局部最优解, 因此将使目标函数 $F\left(x_{i}\right.$, $\left.m_{i}, f_{\mathrm{c}}^{\prime}, f_{\mathrm{c}}\right)^{*}$ 达到最大的全局最优解记为 $\left[\left(x_{I}\right.\right.$, $\left.\left.m_{I}, f_{\mathrm{c}}^{\prime}, f_{\mathrm{c}}\right)^{*}\right]^{*}$. 可见, 全局最优解就是在局部最 优解集合中选择最优的一个.

借鉴极点搜索算法思想设计的新算法步骤为:

1) 初始化参数, 并将当前的最优解记作 [ $\left(x_{I}\right.$, $\left.\left.m_{I}, f_{\mathrm{c}}^{\prime}, f_{\mathrm{c}}\right)^{*}\right]^{*}=(0,0,0,0)$.

2) 依据下层 $I$ 个从者规划的相关性, 生成下层 规划可行的顶点组合集, 并存储于 $I \times N$ 的矩阵中. 其中, $I$ 为客户数量; $N$ 为下层可行顶点组合数, 设 置 $n=1$.

$3)$ 取矩阵 $n$ 列 $\left(x_{i}^{n}, m_{i}^{n}\right)$ 作为下层最优解, 计算 其映射的上层决策变量的可行域 $Q_{n}$.
4) 求解 $\left(x_{i}, m_{i}\right)=\left(x_{i}^{n}, m_{i}^{n}\right),\left(f_{\mathrm{c}}^{\prime}, f_{\mathrm{c}}\right) \in Q_{n}$ 的 规划问题, 并将 $n$ 更新为 $n+1$.

5) 据步骤4) 结果判断其有无最优解, 若有, 继续步骤 6), 否则, 转向步骤 7).

6) 判断此最优解 $\left(x_{I}, m_{I}, f_{\mathrm{c}}^{\prime}, f_{\mathrm{c}}\right)$ * 是否优于当 前最优解 $\left[\left(x_{I}, m_{I}, f_{\mathrm{c}}^{\prime}, f_{\mathrm{c}}\right)^{*}\right]^{*}$, 若是, 则将 $\left(x_{I}\right.$, $\left.m_{I}, f_{\mathrm{c}}^{\prime}, f_{\mathrm{c}}\right)^{*}$ 赋值于 $\left[\left(x_{I}, m_{I}, f_{\mathrm{c}}^{\prime}, f_{\mathrm{c}}\right)^{*}\right]^{*}$; 否则, 转至步骤 7).

$7)$ 若 $n>N$, 则结束当前的运算, [ $\left(x_{I}, m_{I}, f_{\mathrm{c}}^{\prime}\right.$, $\left.\left.f_{\mathrm{c}}\right)^{*}\right]^{*}$ 为全局最优解; 否则, 返回步骤 3$)$.

\section{4 结果与分析}

算例搜索过程如图 1. 其中, $x$ 为深层服务的定 价; $y$ 为基础服务的定价; $z$ 为提供商的利润额, 最 优解位于分布的最高处, 此时客户 1 选择购买深层 服务, 其余客户选择购买基础服务, 云提供商利润 达 $21.81 \times 10^{6}$ 美元/年, 上下层规划所对应的决策
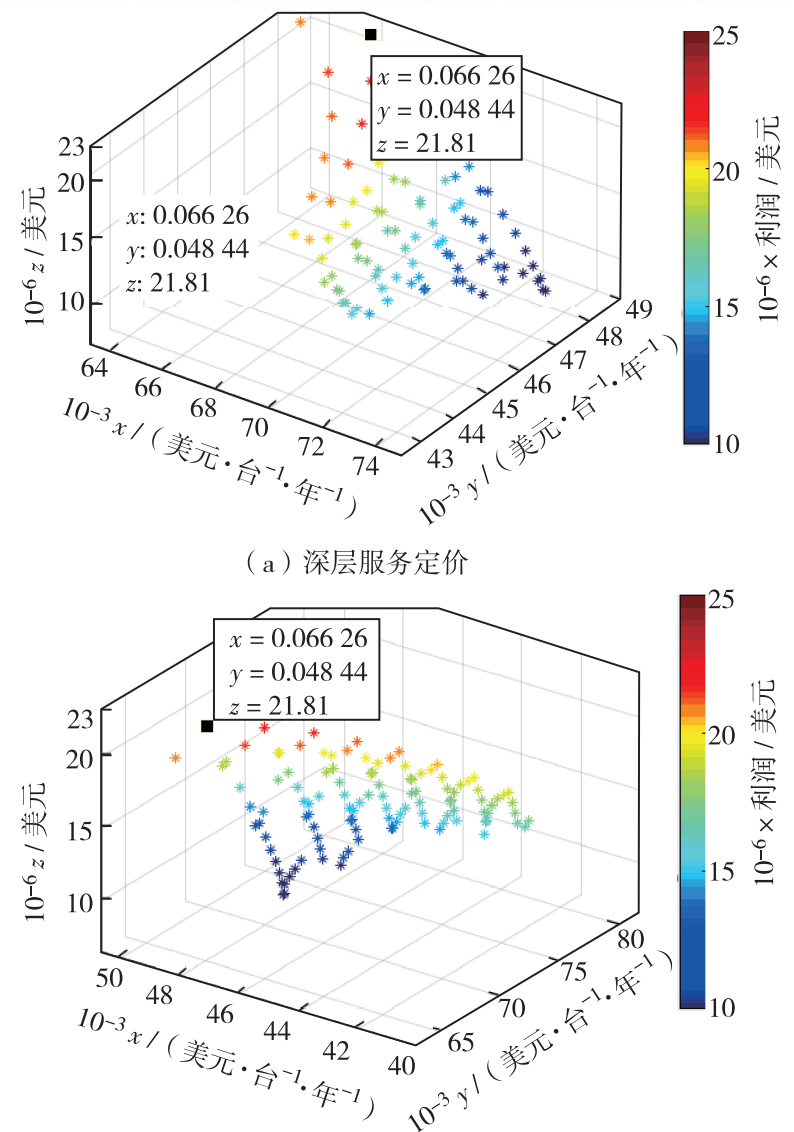

(b) 基础服务定价

图 1 搜索过程中最优点分布

Fig. 1 (Color online) The optimal solution distribution in the search process 
值请扫描论文末页右下角二维码见表 S1.

在搜索过程中，提取客户数量与提供商最优利 润额的变化趋势如图 2. 由图 2 可见，提供商利润 随客户数量增加而增大，这契合了云服务企业固定 成本高和边际成本低的特征，且提供商定价在满足 所有潜在客户要求的过程中, 自身利润也会提高.

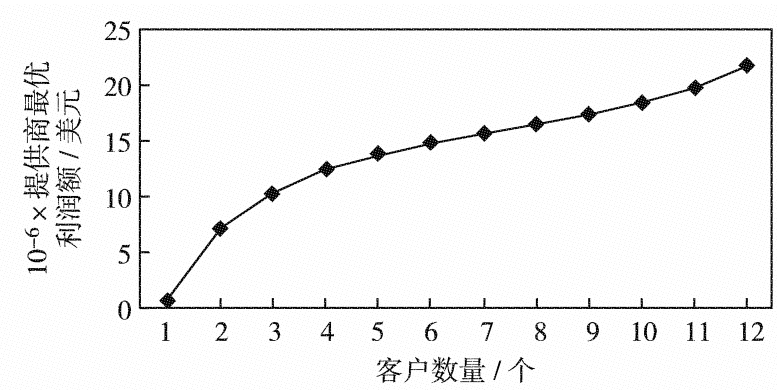

图 2 提供商最优利润额与客户数量关系图

Fig. 2 The relationship between the supplier's optimal profit and the number of customers

购买服务类型不同会导致提供商利润波动，提 取全部客户购买服务时的搜索过程，绘制出服务类 型客户量与提供商利润曲线，如图 3. 由图 3 可见， 定价曲线在 $11 \sim 12$ 个时出现急速下降, 收费价格 从 $6.62 \times 10^{4}$ 美元/台降至 $6.37 \times 10^{4}$ 美元/台，表 明若满足首位客户 (最大客户) 购买深层服务的要 求，会迫使定价大幅下调，导致利润商总利润减 少. 调节定价策略，让首位客户购买基础服务类 型，保留其他 11 位客户深层服务的决策选择，可 使提供商的利润达到最优.

这一现象的机理在于大客户的企业规模大，设 备数量多, 规模效应使设备监控与维修人员得到了

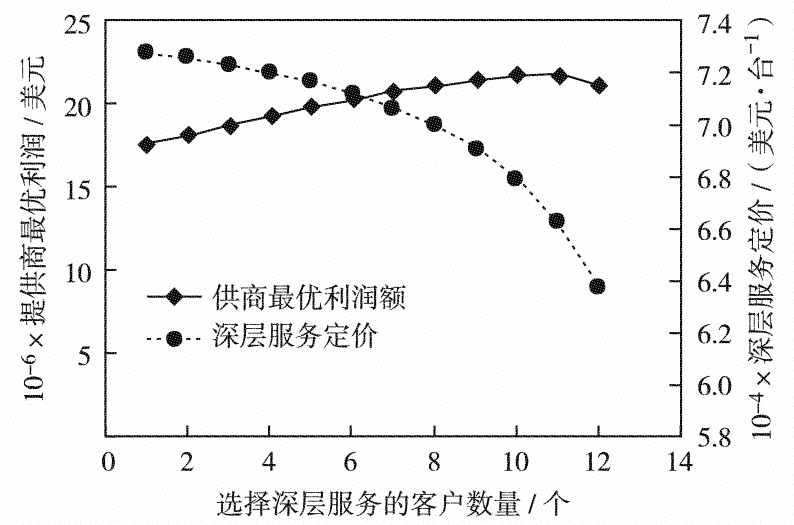

图 3 客户选择服务类型与提供商最优利润额关系

Fig. 3 (Color online) The relationship between the type of service selected by the customer and the optimal profit of the service supplier
有效利用，对通过平台实现企业间设备监控维修资 源共享外包的需求减少，因而倾向选择基础服务类 型. 若要使最大客户接受深层服务, 则需调低深层 服务价格，使其有足够的动力将设备监控维修服务 外包，最终导致总利润下降.

\section{结 语}

1）云监控平台服务进一步扩展的方向包括： 一是绿色制造的发展方向，通过增加采集的数据类 型，为关键设备或大能耗设备安装智能电表、水表 等能耗计量装置，并采集智能电表的三相电流数 据、水表的流量等，通过平台对能耗进行监控，挖 掘节能的生产管理措施，降低企业能耗与制造成 本; 二是智能制造的发展方向，增加对生产工艺参 数的数据采集，将能对客户工厂的生产过程进行智 能监控，为客户提供生产优化建议，提高生产率， 降低质量成本，可根据生产优化服务内容的深度等 级进行收费，或根据所产生的效益的百分比进行收 费; 三是支持制造资源的动态整合，在云制造场景 下，设备加工完成的工件将由自动导引车 ( automatic guided vehicle, AGV) 群协同实现自动运输, 各类生 产设备、能源与员工的云监控统一调度是未来的发 展方向. 在云制造模式下，用户通过智能制造服务 平台便能随时随地按需获取全生命周期活动中的各 类软、硬件制造资源与制造能力 ${ }^{[16]}$.

2) 本研究的研究结果支持经营模式的创新. 本研究提出的平台服务收费博弯分析与双层随机规 划模型可以推广应用于设备租赁服务收费问题，推 动新型生态系统的形成，支持系统运营从设备产品 销售模式转向设备租赁服务模式的转变，在新的模 式以及平台实时监测的支持下，客户可按使用设备 进行高质量生产的时间长度交纳设备租凭费，这将 提高设备供应商改善设备质量、延长设备使用寿命 的积极性，最终降低客户的设备成本，提高生产质 量与安全水平，实现产业链总利润最大化.

3 ）云服务作为新业态出现的时间虽然不久, 但已是商界关注的焦点，云服务类型也迅速朝着多 样化趋势发展，但至今没有形成统一的定价标准. 本研究分析了云监控服务提供商与客户的博弯特 征, 以利润为目标函数，综合考虑了现实中的不确 定因素，构建出双层随机规划定价模型，并通过具 体的实例，验证模型的有效性. 通过分析计算结果 发现在云服务行业中，客户量的提高势必会带来利 
润的增加，同时多类型的服务模式在一定程度上有 助于服务提供商利润的增加.

但是，本研究模型计算出的最优状态，会出现 部分客户最优利润值较低的现象，而利润吸引度的 缺乏，可能导致客户临时更改策略，从而影响提供 商的最优利润．针对这些客户使用价格歧视策略降 低收费，或者使用其他方法来最低成本实现最优状 态稳定性的提升，是后续研究的方向.

基金项目：广东省自然科学基金资助项目（2018A030313079）; 广 东省软科学资助项目 (2019A101002006); 广州市哲学 社会科学发展 “十三五” 规划资助项目 (2018GZYB16)

作者简介：赖朝安 $(1973-)$, 华南理工大学副教授、博士. 研究 方向：智能制造和技术预见. E-mail: chalai@ scut.edu.cn

引 文: 赖朝安, 侯延行. 基于双层随机规划的云监控平台定价 策略 $[\mathrm{J}]$. 深圳大学学报理工版, 2020, 37 (4)：433440 .

\section{参考文献 / References:}

[ 1 ] SENYO P. K, ADDAE E, BOATENG R. Cloud computing research: a review of research themes, frameworks, methods and future research directions $[\mathrm{J}]$. International Journal of Information Management, 2018, 38(1) : 128139.

[ 2 ] YOUSEFF L, BUTRICO M, SILVA D D. Toward a unified ontology of cloud computing [C] // Grid Computing Environments Workshop. Austin, USA: IEEE, 2009: 110.

[ 3 ] CHUN S H, CHOI B S. Service models and pricing schemes for cloud computing $[\mathrm{J}]$. Cluster Computing, 2014, 17(2) : 529-535.

[4] 吴 俊, 徐 溟. 公有云服务计费模式比较研究 $[\mathrm{J}]$. 电信科学, 2012, 28(1): 127-132.

WU Jun, XU Ming. A Comparative analysis on the billing model of public cloud service $[\mathrm{J}]$. Telecommunications Science, 2012, 28(1) : 127-132. (in Chinese)

[ 5 ] MIHAILESCU M, TEO Y M. Dynamic resource pricing on federated clouds $[\mathrm{C}] / /$ IEEE/ACM International Conference on Cluster, Cloud and Grid Computing. Melbourne, Australia: IEEE Computer Society, 2010: 513-517.

[ 6 ] YEO C S, VENUGOPAL S, CHU Xingchen, et al. Autonomic metered pricing for a utility computing service $[\mathrm{J}]$. Future Generation Computer Systems, 2010, 26 ( 8 ): 1368-1380.

[ 7 ] SHARMA B, THULASIRAM R K, THULASIRAMAN P, et al. Clabacus: a risk-adjusted cloud resources pricing model using financial option theory $[\mathrm{J}]$. IEEE Transactions on Cloud Computing, 2015, 3(3) : 332-344.
[ 8 ] HU Zhineng, YAN Shiyu, LV Chengwei, et al. Sustainable development oriented bi-level dynamic programming method toward the coal-water conflict in China $[\mathrm{J}]$. Energy \& Environment, 2019, 30(8) : 1396-1436.

[ 9 ] ZHANG Fan, ENGEL B A, ZHANG Chenglong, et al. Agricultural production planning approach based on interval fuzzy credibility-constrained bi-level programming and Nerlove supply response theory $[\mathrm{J}]$. Journal of Cleaner Production, 2019, 233 : 1158-1169.

[10] KARIMI H, JADID S, SABOORI H. Multi-objective bi-level optimisation to design real-time pricing for demand response programs in retail markets $[\mathrm{J}]$. IET Generation, Transmission \& Distribution, 2019, 13(8) : 1287-1296.

[11] BENITA F, LOPEZ-RAMOS F, NASINIS. A bi-level programming approach for global investment strategies with financial intermediation $[\mathrm{J}]$. European Journal of Operational Research, 2019, 274(1) : 375-390.

[12 ] AXTELL R L. Zipf distribution of U. S. firm sizes [J]. Science, 2001, 293(5536): 1818.

[13 ] HUANG Zhaoke, YANG Chunhua, ZHOU Xiaojun, et al. A novel cognitively inspired state transition algorithm for solving the linear bi-level programming problem $[\mathrm{J}]$. Cognitive Computation, 2018, 10(1) : 816-826.

[14] LACHHWANI K C. On solving multi-objective linear bi-level multi-follower programming problem $[\mathrm{J}]$. International Journal of Operational Research, 2018, 31(4): 442-460.

[15] CANDLER W, TOWNSLEY R. A linear two-level programming problem $[\mathrm{J}]$. Computers \& Operations Research, 1982, 9(1) : 59-76.

[16] 李伯虎，柴旭东，侯宝存，等. 云制造系统 3.0一种 “智能 + ” 时代的新智能制造系统 $[\mathrm{J}]$. 计算机 集成制造系统，2019，25(12)：2997-3012.

LIBohu, CHAI Xudong, HOU Baocun, et al. Cloud manufacturing system 3. 0 : new intelligent manufacturing system in era of “intelligence + " $[\mathrm{J}]$. Computer Integrated Manufacturing Systems, 2019, 25 ( 12 ) : 2997 3012. (in Chinese)

【中文责编：英 子; 英文责编: 木 柯】 